2. We may also, by reference to the pedal line, find the equation to the circum-circle of a triangle formed by three lines the equations to which are given in Cartesian co-ordinates.

We have to express analytically the fact that the area of the triangle formed by joining the feet of perpendiculars on the sides of the given triangle from the point $(x, y)$ is zero, since it becomes a pedal line of the given triangle.

If, therefore, $\mathrm{DEF}$ be the pedal line of the triangle $\mathrm{ABC}$ corresponding to the point $P$, we have

$$
\Delta \mathrm{PFE}+\triangle \mathrm{PDE}-\triangle \mathrm{PFD}=0,
$$

that is,

$$
\mathrm{PF} \cdot \mathrm{PE} \sin \mathrm{A}+\mathrm{PD} \cdot \mathrm{PEsin} \mathrm{C}-\mathrm{PF} \cdot \mathrm{PD} \sin \mathrm{B}=0 \text {; }
$$

so that, if the equations to the sides of the triangle $\mathrm{ABC}$ be of the form $x \cos a+y \sin a-p=0$, the equation to the circum-circle is

$$
\begin{gathered}
\left(x \cos \alpha_{2}+y \sin \alpha_{2}-p_{2}\right)\left(x \cos \alpha_{3}+y \sin \alpha_{3}-p_{3}\right) \sin \left(\alpha_{2}-\alpha_{3}\right) \\
\text { + two similar terms }=0 .
\end{gathered}
$$

If the equations to the sides of the triangle be given in the form $a x+b y+c=0$, the equation to the circum-circle is

$$
\begin{gathered}
\left(a_{2} b_{3}-a_{3} b_{3}\right)\left(a_{1}^{2}+b_{1}^{2}\right)\left(a_{2} x+b_{2} y+c_{2}\right)\left(a_{3} x+b_{3} y+c_{3}\right) \\
+ \text { two similar terms }=0 .
\end{gathered}
$$

[We may also show absolutely that this equation does represent the circum-circle. For the locus represented by it goes through $\mathrm{A}, \mathrm{B}, \mathrm{C}$, since the co-ordinates of each obviously satisfy the equation; and, further, the equation represents a circle, since on examination it will be found that the coefficients of $x^{2}$ and $y^{2}$ are equal, while that of $x y$ is zero.

The pedal line of a triangle and theorems connected with it.

By George A. Gibson, M.A

Most of the following theorems occur in a more or less explicit form in text-books on the geometry of the parabola; but it will not, I hope, be without interest and value to consider then independently, and to prove them by using only the propositions of Euclid.

1. If the perpendiculars $\mathrm{AD}, \mathrm{BE}, \mathrm{CF}$ of a triangle $\mathrm{ABC}$ are produced to meet the circumcircle of the triangle in $\mathrm{X}, \mathrm{Y}, \mathrm{Z}$ respec- 
tively; and if through the orthocentre $O$ any line be drawn meeting the sides $\mathrm{BC}, \mathrm{CA}, \mathrm{AB}$ in $\mathrm{U}, \mathrm{V}, \mathrm{W}$ respectively, then $\mathrm{XU}, \mathrm{YV}, \mathrm{ZW}$ intersect the circumcircle at the same point $S$, the pedal of which is parallel to the line through $O$ and is midway between $S$ and that line.

From the orthocentre $O$ (fig. 26) draw any line OU cutting BC at $\mathrm{U}$ and let $\mathrm{XU}$ meet the circumcircle at $\mathrm{S}$.

Draw SL perpendicular to $\mathrm{BC}$, meeting $\mathrm{OU}$ at $\mathrm{P}$.

Assume the property that $\mathrm{OD}$ is equal to $\mathrm{DX}$.

Then since the triangle $\mathrm{ODU}, \mathrm{XDU}$ are congruent, so are the triangles $S L U, P I U$ and therefore $S L=L P$.

Also $\angle \mathrm{UOX}=\angle \mathrm{UXO}=\angle \mathrm{SCA}$.

Similarly it may be shown that if SY meet CA in V and if SM be drawn perpendicular to $C A$ and produced to meet $O V$ at $Q$, then $\mathrm{SM}=\mathrm{MQ}$ and $\angle \mathrm{VOY}=\angle \mathrm{SCB}$.

Hence $\angle \mathrm{UOX}+\angle \mathrm{VOY}=\angle \mathrm{ACB}=$ supplement of $\angle \mathrm{XOY}$.

Therefore $O U$ and $O V$ are in the same straight line.

Similarly it may be shown that $\mathrm{ZS}$ meets $\mathrm{AB}$ at $\mathrm{W}$, the point of intersection of $\mathrm{UO}$ and $\mathrm{AB}$, and that if $\mathrm{SN}$ be drawn perpendicular to $A B$ and produced to meet $U W$ at $R$, then $S N=N R$.

$\mathrm{L}, \mathrm{M}, \mathrm{N}$ being the middle points of SP, SQ, SR respectively, are therefore collinear, and obviously LM bisects SO.

2. If on the pedal line a point $G$ be taken (fig. 27) and if through $G$ a line be drawn perpendicular to $S G$ meeting the sides $B C, C A$, $\mathrm{AB}$ in $\mathbf{A}^{\prime}, \mathrm{B}^{\prime}, \mathrm{C}^{\prime}$ respectively, then $\mathbf{S A}^{\prime}, \mathbf{S B}^{\prime}, \mathbf{S C}^{\prime}$ make equal angles with the sides $\mathrm{BC}, \mathrm{CA}, \mathrm{AB}$.

The points $S, G, L, A^{\prime}$ are concyclic, since the angles $S G A^{\prime}$, SLA' $A^{\prime}$ are right angles. Hence the angle $S A^{\prime} L$ is equal to the angle SGN. In the same way it may be shown that the angles $\mathbf{S B}^{\prime} \mathbf{M}$, $\mathrm{SC}^{\prime} \mathrm{N}$ are each equal to the angle $\mathrm{SGN}$ and therefore to the angle SA'L.

It is easy to show, conversely, that if $A^{\prime}, B^{\prime}, C^{\prime}$ are three collinear points on the sides such that the angles $\mathbf{S A}^{\prime} \mathbf{B}, \mathbf{S B}^{\prime} \mathbf{A}, \mathbf{S C}^{\prime} \mathbf{A}$ are equal, then $S$ lies on the circumcircle of the triangle and the pedal line of $S$ meets $A^{\prime} B^{\prime}$ at $G$ so that $S G$ is perpendicular to $A^{\prime} B^{\prime}$.

3. If in fig. $27, \mathrm{~A}^{\prime} \mathrm{B}^{\prime} \mathrm{C}^{\prime}$ be any line meeting the sides of the triangle in $A^{\prime}, B^{\prime}, C^{\prime}$, then it is readily shown that the circumcircles of the four triangles formed by the four lines, $\mathrm{AB}, \mathrm{BC}, \mathrm{CA}, \mathrm{A}^{\prime} \mathbf{B}^{\prime}$ 
intersect in some point $\mathbf{S}$. All four triangles have obviously the same pedal line with respect to $\mathrm{S}$. Hence, if $\mathrm{O}$ be the orthocentre of the triangle $\mathrm{ABC}$, it follows from (1) that the orthocentres of all four triangles lie on the line through $O$ parallel to the pedal line of $\mathrm{S}$.

It is also readily seen that the lines joining $S$ to the points in which any one of the four lines cuts the other three are equally inclined to these three.

4. In fig. 26 it is not necessary that $O$ should be the orthocentre in order that $\mathrm{XU}, \mathrm{YV}, \mathrm{ZW}$ should intersect the circumcircle of the triangle at the same point; all that is necessary is that $\mathrm{AX}, \mathrm{BY}$, $\mathrm{C} Z$ should be concurrent at the point $O$ through which the line UVW is drawn.

The converse is also true, viz., if any line cut the sides at $U, V$, $W$ and if any point $S$ on circumcircle be joined to $U, V, W$ and produced to meet the circumference at $\mathrm{X}, \mathrm{Y}, \mathrm{Z}$, then $\mathrm{AX}, \mathrm{BY}, \mathrm{CZ}$ will be concurrent at some point $O$ on the line UVW.

The proof is, in both cases, given immediately by Pascal's Theorem. Thus if, as in (1), we suppose OU drawn, XU produced to $S$ and $S Y$ joined cutting $C A$ in $V$, then applying Pascal's Theorem to the hexagon AXSYBC we see that AX, YB intersect at $O, X S$, $\mathrm{BC}$ at $\mathrm{U}, \mathrm{SY}, \mathrm{CA}$ at $\mathrm{V}$ and therefore $\mathrm{V}$ lies in $\mathrm{UO}$ produced. Similarly it may be shown that $W$ lies in UO produced.

It may be noticed that if the line be the pedal line of the point in the converse theorem, then the point of concurrence is the point at infinity on the line. Thus if in fig. 26, SL meet the circle at $x$, then $A x$ is parallel to $\mathrm{LM}$, for

$\angle \mathrm{S} x \mathrm{~A}=\angle \mathrm{SBA}=\angle \mathrm{SLN}$ since SBLN is a cyclic quadrilateral.

The figure $\mathrm{AxPO}$ is therefore a parallelogram; $x \mathrm{P}$ is equal to $\mathrm{AO}$ and the mid point of AP, being also the mid point of $\mathrm{Ox}$, is on the nine-point circle of the triangle.

5. If $A^{\prime} C^{\prime} B^{\prime}$ be any line drawn according to the construction of paragraph (2), then the ratio of the intercepts $A^{\prime} C^{\prime}, C^{\prime} B^{\prime}$ is constant, and also the ratios $\mathrm{BC}^{\prime}: \mathrm{CB}^{\prime}, \mathrm{CA}^{\prime}: \mathrm{AC}^{\prime}, \mathrm{AB}^{\prime}: \mathrm{BA}^{\prime}$ : for (fig. 27)

the triangles $A^{\prime} C^{\prime} S$, LNS are similar

$\therefore A^{\prime} \mathbf{C}^{\prime}: \mathbf{C}^{\prime} \mathbf{S}=\mathrm{LN}: \mathrm{NS}$. 
In the same way from the triangles $\mathrm{SC}^{\prime} \mathrm{B}^{\prime}$, SNM

$$
\begin{aligned}
C^{\prime} S: C^{\prime} B^{\prime} & =N S: N M \\
\therefore \quad A^{\prime} C^{\prime}: C^{\prime} B^{\prime} & =L N: N M \text { a constant ratio. }
\end{aligned}
$$

Again, the triangles $\mathrm{BC}^{\prime} \mathrm{S}, \mathrm{CB}^{\prime} \mathrm{S}$ are equiangular, for

$$
\begin{gathered}
\angle \mathrm{SBC}^{\prime}=\angle \mathrm{SCB}^{\prime} \text { and } \angle \mathrm{SC} \mathrm{C}^{\prime} \mathrm{B}=\angle \mathrm{SB}^{\prime} \mathrm{C} \\
\therefore \mathrm{BC}^{\prime}: \mathrm{CB}^{\prime}=\mathrm{BS}: \mathrm{CS} \text {. }
\end{gathered}
$$

Similarly $\mathbf{C A}^{\prime}: \mathrm{AC}^{\prime}=\mathrm{CS}: \mathrm{AS}, \mathrm{AB}^{\prime}: \mathrm{BA}^{\prime}=\mathrm{AS}: \mathrm{BS}$.

Further if any other point $H$ were taken on $L M$ and a line drawn through $\mathbf{H}$ perpendicular to $\mathrm{SH}$ and cutting $\mathbf{B C}, \mathbf{C A}, \mathbf{A B}$, $\mathrm{B}^{\prime} \mathrm{C}^{\prime}$ in $\mathrm{T}, \mathrm{U}, \mathrm{V}, \mathrm{W}$, then if the first four lines be fixed while $\mathrm{TW}$ varies, the ratios $U V: V W$ and $U T: W T$ are constant so that the cross ratio of the points $T, U, V, W$ is constant and the ratio of the three intercepts UV, VW, WT is constant.

6. It is obvious that the properties deduced in paragraph 5 are those of tangents to a parabola with focus $S$. It might be easily shown, for example, that when $A^{\prime} B^{\prime}$ is consecutive to $B C$, the point $A^{\prime}$ is such that $A^{\prime} B: B C$ is equal to $L N: N M$ and $\angle S A^{\prime} L=\angle S L N$. Many of the properties of the parabola when it is determined by means of its tangents are very easily deduced by constructing the triangle formed by three of them and proceeding as in 5 .

Thus, given four tangents, draw the tangents which pass through a given point.

The second point, $\mathrm{S}$, in which the circumcircles of any two triangles formed by the four tangents intersect is the focus and the pedal line of $S$ is the tangent at the vertex. If on the line joining $S$ to the given point $P$ a circle be described cutting the pedal line in $Q$ and $R, P Q$ and $P R$ are the tangents.

Or again, if on the sides $A B, A C$ of a triangle (fig. 27) two points $B^{\prime}, C^{\prime}$ be taken such that $B^{\prime}: C^{\prime}=$ constant, then the envelope of $B^{\prime} C^{\prime}$ is a parabola inscribed in the triangle and the locus of any point $I^{\prime}$ dividing $B^{\prime} C^{\prime}$ in a given ratio is the (second) tangent through $\mathrm{I}$ where $\mathrm{BI}: \mathrm{IC}=$ given ratio.

7. As another application to the parabola, I had selected the theorem that the centre of every triangle self-polar with respect to a parabola lies on the directrix. The proof required the theorem that the circles on the three diagonals of a complete quadrilateral have a common radical axis, namely the line containing the four 
orthocentres of the four triangles determined by the four sides of the quadrilateral. I reached a proof that seemed to leave nothing to be desired in point of simplicity ; but I have since found that it is practically the same as that given in Townsend's Mod. Geont., vol. i., p, 253, and I have therefore struck it out.

It may be noted, however, that this elementary demonstration gives at once the theorem on the self-conjugate triangle, for the circle circumscribing the diagonal triangle cuts the circles on the three diagonals orthogonally and hence its centre must lie on the radical axis of the circles, i.e., on the directrix of the parabola which is inscribed in the quadrilateral. Further the line of collinearity of the mid-points of the three diagonals must be parallel to the axis of the parabola.

8. Several of the ordinary standard theorems on the parabola are readily deducible as special cases of the properties of the complete quadrilateral mentioned in the preceding paragraph.

(i) Let, in fig. 27, $\mathrm{AB}, \mathrm{A}^{\prime} \mathrm{B}^{\prime}$ become consecutive to $\mathrm{CA}, \mathrm{BC}$ respectively and suppose their points of contact $b, a$. Then the diameter of the quadrilateral becomes the line through $\mathrm{C}$ and the mid-point of the chord $a b$ and by the theorem of (7) this line is parallel to the axis of the parabola.

(ii) Again, let $\mathrm{AB}$ remain while $\mathrm{A}^{\prime} \mathrm{B}^{\prime}$ becomes consecutive to $B C$, then the diameter of the quadrilateral becomes the line through the mid point of $\mathrm{BO}$ and the mid point of $\mathrm{Aa}$. Hence if any triangle circumscribe a parabola, the mid point of any side and the mid point of the line from the point of contact of that side to the opposite vertex are on a line parallel to the axis of the parabola.

(iii) Suppose now the tangent AB (fig. 27) bisected at its point of contact $c$, then by (ii) $c$ and the mid point of $\mathrm{C} c$ are on a line parallel to the axis, i.e. $\mathrm{C} c$ is parallel to the axis. Hence by (i) $\mathrm{C}$, $c$, and the mid point of the chord of contact $a b$ are on a line parallel to the axis. In other words, any point $c$ on the parabola, the mid point of any chord parallel to the tangent at $c$ and the point of intersection of the tangents at the ends of the chord are on a line parallel to the axis-the fundamental property of a diameter. By the principle of paragraph (5), A, B are the mid points of $c b, c a$ when $c$ is the point of $\mathrm{AB}$ and therefore $\mathrm{C} c=c d$ if $d$ be the midpoint of $a b$.

(iv) When $\mathrm{AB}, \mathrm{A}^{\prime} \mathrm{B}^{\prime}$ become consecutive to $\mathrm{CA}, \mathrm{BC}$ as (i), the 
circles on $\mathrm{BB}^{\prime}, \mathrm{CC}^{\prime}$ as diameters become the point circle $\mathrm{C}$, and the directrix is by (7) the radical axis for the circle on $a b$ as diameter and the point circle $\mathrm{C}$. Hence if $\mathrm{C}$ be on the directrix the circle on the chord $a b$ must touch it at $\mathrm{C}$ and therefore the tangents from a point on the directrix are at right angles.

(v) Again taking $d$ as the mid point of the chord $a b$ and assuming that $a d^{2}$ varies as $\mathbf{C} d$, where $\mathbf{C}$ is the point of intersection of tangents at ends of $a, b$, then the distance of $d$ from the image of $\mathrm{O}$ in the directrix will be constant for all tangents drawn from a point on the same diameter.

For the image of $\mathrm{C}$, say $e$, is the second limiting point for the system $\mathrm{C}$ and circle on $a b$. Hence $d e . d \mathrm{C}=a b^{2} ; \therefore d e$ is constant.

Eighth Meeting, June 12, 1891.

R. E. Allardice, Esq., M.A., F.R.S.E., President, in the Chair.

\section{On a Representation of Elliptic Integrals by Curvilinear} Aros.

By John M'Cowan, M.A., B.Sc.

It is well known that the elliptic integral of the second kind may be represented by the arc of an ellipse, and mathematicians have sought with various success to represent similarly by the arc of an algebraic curve the elliptic integral of the first kind. The general solution of the problem has not been obtained, but Serret and Cayley have given solutions of a very general character.

If, however, the condition that the curve be algebraic be not imposed solutions may be obtained without difficulty. That here given has the peculiarity that the set of elliptic integrals of the first kind for all values of the modulus is represented by the arcs of one system of curves, in several distinct ways, each of a very simple geometric character. The system of curves is further interesting, as representing in the different groupings which naturally arise different systems of stream lines due to vortices in two dimensional fluid motion. 